\title{
Dependence of Eigenvalues of a Class of Higher-Order Sturm-Liouville Problems on the Boundary
}

\author{
Qiuxia Yang, ${ }^{1}$ Wanyi Wang, ${ }^{2}$ and Xingchao Gao ${ }^{3}$ \\ ${ }^{1}$ Information Management College, Dezhou University, Dezhou 253023, China \\ ${ }_{2}^{2}$ Mathematics Science College, Inner Mongolia University, Huhhot 010021, China \\ ${ }^{3}$ Automobile Engineering College, Dezhou University, Dezhou 253023, China
}

Correspondence should be addressed to Qiuxia Yang; 1072003484@qq.com

Received 11 March 2014; Accepted 9 September 2014

Academic Editor: Sellakkutti Rajendran

Copyright (C) 2015 Qiuxia Yang et al. This is an open access article distributed under the Creative Commons Attribution License, which permits unrestricted use, distribution, and reproduction in any medium, provided the original work is properly cited.

\begin{abstract}
We show that the eigenvalues of a class of higher-order Sturm-Liouville problems depend not only continuously but also smoothly on boundary points and that the derivative of the $n$th eigenvalue as a function of an endpoint satisfies a first order differential equation. In addition, we prove that as the length of the interval shrinks to zero all $2 k$ th-order Dirichlet eigenvalues march off to plus infinity; this is also true for the first (i.e., lowest) eigenvalue.
\end{abstract}

\section{Introduction}

Dauge and Helffer in $[1,2]$ considered the second-order Sturm-Liouville (SL) problems and obtained the equations for the eigenvalues of self-adjoint separated boundary conditions. In addition, they showed that the lowest Dirichlet eigenvalue is a decreasing function of the endpoints and thus must have a finite or infinite limit as the end-points approach each other but left open the question of whether this limit is finite or infinite. In [3] the authors showed that it is infinite.

Following the above, Ge et al. in [4] considered the fourth-order Sturm-Liouville differential equation

$$
\left(p_{0} y^{\prime \prime}\right)^{\prime \prime}+p_{2} y=\lambda w y
$$

with $p_{0}, p_{2}, w: I=(A, B) \rightarrow \mathbb{R}, 1 / p_{0}, p_{2} \in L_{\mathrm{loc}}(I)$ and $w>$ 0 a.e. on $I$. They showed that its Neumann eigenvalues and Dirichlet eigenvalues, as functions of an endpoint, satisfy the same differential equation form as $[1,2]$ and the equation for the eigenvalues of self-adjoint separated boundary conditions

$$
\lambda^{\prime}=-\frac{1}{p_{0}}\left(p_{0} u^{\prime \prime}\right)^{2}+\left(p_{2}-\lambda w\right) u^{2}+2\left(p_{0} u^{\prime \prime}\right)^{\prime} u^{\prime} .
$$

In particular, they also proved that the lowest Dirichlet eigenvalue is a decreasing function of the endpoints and thus have infinite limit as the endpoints approach each other.
In this paper, partly motivated by the work of Ge et al. in [4], we continue to consider the dependence of eigenvalues of more general form and higher $2 k$ th-order Sturm-Liouville problems on the boundary and also show that the eigenvalues depend not only continuously but also smoothly on boundary points and that the $2 k$ th-order Dirichlet eigenvalues, as functions of the endpoint $b$, satisfy a differential equation of the form

$$
p_{0} \lambda^{\prime}=-\left(u^{[k]}\right)^{2} .
$$

We also find the equation satisfied by the $2 k$ th-order Neumann eigenvalues

$$
\lambda^{\prime}=\sum_{r=1}^{k-1} p_{k-r}\left(u^{(r)}\right)^{2}+\left(p_{k}-\lambda w\right) u^{2}
$$

and the equation for the eigenvalues of self-adjoint separated boundary conditions,

$$
\begin{aligned}
\lambda^{\prime}= & \sum_{r=1}^{k-1}\left[p_{k-r}\left(u^{(r)}\right)^{2}+2 u^{[2 k-r]} u^{(r)}\right] \\
& +\left(p_{k}-\lambda w\right) u^{2}-\frac{\left(u^{[k]}\right)^{2}}{p_{0}} .
\end{aligned}
$$


Furthermore, we prove that as the length of the interval shrinks to zero all higher $2 k$ th-order Dirichlet eigenvalues march off to plus infinity; this is also true for the first (i.e., lowest) eigenvalue. Although we use the same method of proof as in [4] to get our main results, the specific process of calculation and proof is not completely the same as in [4]. Besides that our conclusions are more concrete and general, theoretical importance, the dependence of the eigenvalues on the interval is fundamental from the numerical point of view (see, e.g., [1-9]).

In Section 2, we summarize some of the basic results needed later and establish the notation. The main results of fourth-order Sturm-Liouville problem are given in Section 3. In Section 4 , we consider higher $2 k$ th-order Sturm-Liouville problems and obtain more important results. The last section involves some interesting description about Sturm-Liouvilletype boundary value problems.

\section{Notation and Basic Results}

Consider the differential equation

$$
\begin{aligned}
& l y=\sum_{r=0}^{k}(-1)^{r}\left(p_{k-r} y^{(r)}\right)^{(r)}=\lambda w y \\
& \text { on }(A, B),-\infty \leq A<B \leq \infty \text { with } \lambda \in \mathbb{R},
\end{aligned}
$$

where

$$
\begin{aligned}
& p_{0}, p_{i}, w: I=(A, B) \longrightarrow \mathbb{R} ; \\
& \frac{1}{p_{0}}, p_{i} \in L_{\mathrm{loc}}(I), \quad w>0 \quad \text { a.e. on } I, \quad i=1,2, \ldots, k .
\end{aligned}
$$

We introduce the quasi derivatives of a function $y, y^{[j]}$, $j=0,1,2, \ldots, 2 k$ as follows:

$$
\begin{aligned}
y^{[j]} & =y^{(j)}, \quad j=0,1,2, \ldots, k-1, \\
y^{[k]} & =p_{0} y^{(k)}, \\
y^{[j]} & =\left(y^{[j-1]}\right)^{\prime}+(-1)^{j-k} p_{j-k} y^{(2 k-j)}, \\
& j=k+1, k+2, \ldots, 2 k ;
\end{aligned}
$$

then $l$ in (6) may be simply written by

$$
l y=(-1)^{k} y^{[2 k]}=\lambda w y .
$$

In this way, the differential expression $l$ on $I$ is defined for all functions $y$ such that $y^{[0]}, y^{[1]}, \ldots, y^{[2 k-1]}$ exist and are absolutely continuous over compact subintervals of $I$.

Let

$$
J=[a, b], \quad A<a<b<B,
$$

and consider boundary conditions (BC)

$$
C\left(\begin{array}{c}
y(a) \\
y^{[1]}(a) \\
\vdots \\
y^{[2 k-1]}(a)
\end{array}\right)+D\left(\begin{array}{c}
y(b) \\
y^{[1]}(b) \\
\vdots \\
y^{[2 k-1]}(b)
\end{array}\right)=\left(\begin{array}{c}
0 \\
0 \\
\vdots \\
0
\end{array}\right)
$$

where the complex $2 k \times 2 k$ matrices $C$ and $D$ satisfy

The $2 k \times 4 k$ matrices $(C \mid D)$ have full rank,

$$
C E C^{*}=D^{*}, \quad E=\left(\begin{array}{ccccc}
0 & 0 & \cdots & 0 & -1 \\
0 & 0 & \cdots & 1 & 0 \\
\vdots & \vdots & \ddots & \vdots & \vdots \\
0 & -1 & \cdots & 0 & 0 \\
1 & 0 & \cdots & 0 & 0
\end{array}\right) \text {. }
$$

A SL boundary value problem consists of (6) together with boundary conditions (BC) (11). With conditions (7), (10), and (12) it is well known that problem (6), (11) is a regular $2 k$ th-order self-adjoint SL problem which has an infinite but countable number of only real eigenvalues.

From [10], these self-adjoint boundary conditions (11)(12) are divided into three disjoint subclasses: separated, coupled, and mixed. In the separated case, there are many forms for the $2 k$ th-order problems. In this paper, we only study one form of them.

Consider the following boundary conditions (BC):

$$
\begin{gathered}
\cos \alpha y(a)-\sin \alpha y^{[2 k-1]}(a)=0 \\
\cos \alpha y^{[1]}(a)-\sin \alpha y^{[2 k-2]}(a)=0
\end{gathered}
$$

$$
\cos \alpha y^{[k-1]}(a)-\sin \alpha y^{[k]}(a)=0, \quad 0 \leq \alpha<\pi,
$$

$$
\begin{gathered}
\cos \beta y(b)-\sin \beta y^{[2 k-1]}(b)=0 \\
\cos \beta y^{[1]}(b)-\sin \beta y^{[2 k-2]}(b)=0
\end{gathered}
$$

$$
\cos \beta y^{[k-1]}(b)-\sin \beta y^{[k]}(b)=0, \quad 0<\beta \leq \pi .
$$

Here we fix $p_{i}(i=0,1,2, \ldots, k), w$ and the boundary condition (constants), and one endpoint and study the dependence of the eigenvalues and eigenfunctions on the other endpoint.

By a solution of (6) on $I$ we mean a function $y^{[0]}, y^{[1]}$, $\ldots, y^{[2 k-1]} \in A C_{\text {loc }}(I)$ and (6) is satisfied a.e. on $I$. Here $A C_{\text {loc }}(I)$ denotes the set of functions which are absolutely continuous on all compact subintervals of $I$.

It is well known that the $2 k$ th-order SL boundary value problem consisting of (6) together with boundary conditions (BC) (13a)-(13c), (14a)-(14c) is a regular $2 k$ th-order selfadjoint boundary value problem which has an infinite but countable number of only real eigenvalues. If $p_{0} \geq 0$, a.e. on $J=(a, b)$, then the eigenvalues are bounded below and can be ordered to satisfy

$$
-\infty<\lambda_{0} \leq \lambda_{1} \leq \lambda_{2} \leq \cdots, \quad \lambda_{n} \longrightarrow+\infty \quad \text { as } n \longrightarrow+\infty \text {. }
$$


Notation. Let $N_{0}=\{0,1,2, \ldots\}$; for the fourth-order or higher-order Dirichlet and Neumann eigenvalues we use the special notation

$$
\lambda_{n}^{D}=\lambda_{n}(0, \pi ; a, b), \quad \lambda_{n}^{N}=\lambda_{n}\left(\frac{\pi}{2}, \frac{\pi}{2} ; a, b\right) .
$$

By a normalized eigenfunction $u$ of the BVP (6), (13a)(14c), we mean an eigenfunction $u$ that satisfies

$$
\int_{a}^{b}|u|^{2} w=1
$$

For fixed $a$ and fixed boundary condition constants $\alpha, \beta$ we abbreviate the notation to $\lambda_{n}(b)$ and study $\lambda_{n}(b)$ as a function of $b$ for fixed $n \in N_{0}$, as $b$ varies in the interval $(a, B)$.

In the following, we present a continuity result for the eigenvalues and eigenfunctions.

Lemma 1. Let self-adjoint boundary value problems be described as (6), (13a)-(14c). Fix the BC and the endpoint $a$ or $b$. Fix $n \in N_{0}$. Let $\lambda_{n}=\lambda_{n}(b)$ for $b \in(a, B)$. Then

(1) $\lambda_{n}(b)$ is a continuous function of $b$ for $b \in(a, B)$.

(2) If $\lambda_{n}(b)$ is simple for some $b \in(a, B)$ then $\lambda_{n}(b)$ is simple for every $b \in(a, B)$.

(3) There exists a normalized eigenfunction $u_{n}(\cdot, b)$ of $\lambda_{n}(b)$ for $b \in(a, B)$ such that, $\left(u_{n}^{[j]}\right)(\cdot, b)(j=0$, $1,2, \ldots, 2 k-1)$ are uniformly convergent in $b$ on any compact subinterval of $(a, B)$; that is,

$$
u_{n}^{[j]}(\cdot, b+h) \longrightarrow u_{n}^{[j]}(\cdot, b), \quad j=0,1,2, \ldots, 2 k-1,
$$

and this convergence is uniform on any compact subinterval of $(a, B)$.

Proof. See the proof of Theorem 3 in [3].

Lemma 2. Assume $u$ and $v$ are solutions of (6) with $\lambda=\mu$ and $\lambda=\nu$, respectively. Then

$$
\begin{aligned}
{[u, v]_{a}^{b} } & =[u, v](b)-[u, v](a) \\
& =(-1)^{k} \sum_{r=0}^{2 k-1}(-1)^{2 k+1-r} u^{[r]} \bar{v}^{[2 k-r-1]} \\
& =(\mu-v) \int_{a}^{b} u \bar{v} w .
\end{aligned}
$$

Proof. This follows from integration by parts.

Lemma 3. Assume a real valued function $f \in L_{\mathrm{loc}}(A, B)$. Then

$$
\lim _{h \rightarrow 0} \frac{1}{h} \int_{t}^{t+h} f=f(t) \quad \text { a.e. in }(A, B)
$$

Proof. See the proof given in [3].

\section{Eigenvalues of Fourth-Order Sturm-Liouville Problem}

In this section, we obtain the differentiability of the eigenvalues of the fourth-order boundary value problem, establish differential equations satisfied by them, and discuss the behavior of the Dirichlet eigenvalues as functions of the endpoint $b$.

Consider the differential equation

$$
\begin{aligned}
& \left(p_{0} y^{\prime \prime}\right)^{\prime \prime}-\left(p_{1} y^{\prime}\right)^{\prime}+p_{2} y=\lambda w y \\
& \text { on }(A, B),-\infty \leq A<B \leq \infty \text { with } \lambda \in \mathbb{R},
\end{aligned}
$$

where

$$
\begin{aligned}
& p_{0}, p_{1}, p_{2}, w: I=(A, B) \longrightarrow \mathbb{R} ; \\
& \frac{1}{p_{0}}, p_{1}, p_{2} \in L_{\mathrm{loc}}(I), \quad w>0 \text { a.e. on } I .
\end{aligned}
$$

Let $J=[a, b], A<a<b<B$ and consider the following boundary conditions $(\mathrm{BC})$

$$
\begin{gathered}
\cos \alpha y(a)-\sin \alpha y^{[3]}(a)=0, \\
\cos \alpha y^{[1]}(a)-\sin \alpha y^{[2]}(a)=0, \quad 0 \leq \alpha<\pi, \\
\cos \beta y(b)-\sin \beta y^{[3]}(b)=0, \\
\cos \beta y^{[1]}(b)-\sin \beta y^{[2]}(b)=0, \quad 0<\beta \leq \pi,
\end{gathered}
$$

where $y^{[1]}=y^{\prime}, y^{[2]}=p_{0} y^{\prime \prime}, y^{[3]}=\left(p_{0} y^{\prime \prime}\right)^{\prime}-p_{1} y^{\prime}$ are quasiderivative. Fix $p_{0}, p_{1}, p_{2}, w$ and the boundary condition (constants) and one endpoint and study the dependence of the eigenvalues and eigenfunctions on the other endpoint.

Theorem 4 (fourth-order Dirichlet eigenvalue-eigenfunction differential equation). Let (22) hold. Consider the BVP (21), (23a)-(24b), with $0 \leq \alpha<\pi$ and $\beta=\pi$, that is, arbitrary separated conditions at $a$ and the fourth-order Dirichlet conditions at $b$. Using the notation of Section 2 and letting $\lambda=\lambda_{n}$, $u=u_{n}$, we have the following differential equation:

$$
\left(p_{0} \lambda^{\prime}\right)(b)=-\left(u^{[2]}\right)^{2}(b, b) \quad \text { a.e. in }(a, B) \text {. }
$$

In particular, if $p_{0}$ is continuous at $b \in[a, B)$ and $p_{0}(b) \neq 0$, then (25) holds at $b$.

Proof. For small $h$, in (19), choose $k=2, \mu=\lambda(b), v=\lambda(b+$ $h)$, and $u=u(\cdot, b), v=u(\cdot, b+h)$. From (19) and the boundary conditions (23a) $-(24 \mathrm{~b})$, noting that $[u, v](a)=0, u(b, b)=0$ and $u^{\prime}(b, b)=0$, we have

$$
\begin{aligned}
& u^{[3]}(b, b) u(b, b+h)-u^{[2]}(b, b) u^{\prime}(b, b+h) \\
& \quad=[\lambda(b)-\lambda(b+h)] \int_{a}^{b} u(s, b) u(s, b+h) w(s) d s .
\end{aligned}
$$


Since

$$
\begin{aligned}
u(b, b+h) & =u(b, b+h)-u(b+h, b+h) \\
& =-\int_{b}^{b+h} u^{\prime}(s, b+h) d s .
\end{aligned}
$$

By Lemmas 1 and 3, we have

$$
\lim _{h \rightarrow 0} \frac{u(b, b+h)}{h}=-u^{\prime}(b, b)
$$

Also from

$$
\begin{aligned}
u^{\prime}(b, b+h) & =u^{\prime}(b, b+h)-u^{\prime}(b+h, b+h) \\
& =-\int_{b}^{b+h} u^{\prime \prime}(s, b+h) d s \\
& =-\int_{b}^{b+h} \frac{\left(p_{0} u^{\prime \prime}\right)(s, b+h)}{p_{0}(s)} d s,
\end{aligned}
$$

we have

$$
\lim _{h \rightarrow 0} \frac{u^{\prime}(b, b+h)}{h}=-\frac{1}{p_{0}(b)}\left(p_{0} u^{\prime \prime}\right)(b, b) .
$$

And we can obtain

$$
\begin{array}{r}
\int_{a}^{b} u(s, b) u(s, b+h) w(s) d s \longrightarrow \int_{a}^{b} u^{2}(s, b) w(s) d s=1 \\
\text { as } h \longrightarrow 0 .
\end{array}
$$

Plugging (28), (30), and (31) into (26) divided by $h$ and taking the limit as $h \rightarrow 0$, we get (25). The second part of the theorem follows from above.

Theorem 5 (fourth-order Neumann eigenvalue-eigenfunction differential equation). Let (22) hold. Consider the BVP (21), (23a)-(24b), with $0 \leq \alpha<\pi$ and $\beta=\pi / 2$, that is, arbitrary separated conditions at $a$ and the fourth-order Neumann conditions at $b$. Using the notation of Section 2 and letting $\lambda=\lambda_{n}, u=u_{n}$, we have the following differential equation:

$$
\begin{aligned}
\lambda^{\prime}(b)= & p_{1}(b)\left(u^{\prime}(b, b)\right)^{2} \\
& +\left(p_{2}(b)-\lambda(b) w(b)\right) u^{2}(b, b) \text { a.e. in }(a, B) .
\end{aligned}
$$

In particular, if $p_{1}, p_{2}$ and $w$ are continuous at $b \in[a, B)$, then (32) holds at $b$.

Proof. The proof is similar to Theorem 4. For small $h$, we choose $\mu=\lambda(b), \nu=\lambda(b+h)$, and $u=u(\cdot, b), v=u(\cdot, b+h)$.
From (19) and the boundary conditions (23a)-(24b), noting that $[u, v](a)=0, u^{[2]}(b, b)=0$ and $u^{[3]}(b, b)=0$, we have

$$
\begin{aligned}
& u^{\prime}(b, b) u^{[2]}(b, b+h)-u(b, b) u^{[3]}(b, b+h) \\
& \quad=[\lambda(b)-\lambda(b+h)] \int_{a}^{b} u(s, b) u(s, b+h) w(s) d s .
\end{aligned}
$$

$$
\begin{aligned}
u^{[3]}(b, b+h) & u^{[3]}(b, b+h)-u^{[3]}(b+h, b+h) \\
= & -\int_{b}^{b+h}\left(u^{[3]}\right)^{\prime}(s, b+h) d s \\
= & \int_{b}^{b+h}\left[p_{2}(s) u(s, b+h)-\lambda(b+h) u(s, b+h) w(s)\right] d s \\
= & \int_{b}^{b+h} p_{2}(s) u(s, b) d s \\
& +\int_{b}^{b+h} p_{2}(s) u(s, b+h)-p_{2}(s) u(s, b) d s \\
& -\lambda(b+h) \int_{b}^{b+h} u(s, b) w(s) d s \\
& +\lambda(b+h) \int_{b}^{b+h}[u(s, b)-u(s, b+h)] w(s) d s \\
= & \int_{b}^{b+h} p_{2}(s) u(s, b) d s-\lambda(b+h) \int_{b}^{b+h} u(s, b) w(s) d s .
\end{aligned}
$$

By Lemmas 1 and 3 we have

$$
\lim _{h \rightarrow 0} \frac{u^{[3]}(b, b+h)}{h}=\left(p_{2}(b)-\lambda(b) w(b)\right) u(b, b) .
$$

In a similar way, we have

$$
\lim _{h \rightarrow 0} \frac{p_{0} u^{\prime \prime}(b, b+h)}{h}=-\left(p_{0} u^{\prime \prime}\right)^{\prime}(b, b) .
$$

Combining $u^{[3]}(b, b)=\left[\left(p_{0} u^{\prime \prime}\right)^{\prime}-p_{1} u^{\prime}\right](b, b)=0$, we also can get

$$
\lim _{h \rightarrow 0} \frac{p_{0} u^{\prime \prime}(b, b+h)}{h}=-p_{1} u^{\prime}(b, b)
$$

When $h \rightarrow 0$, noting that

$$
\int_{a}^{b} u(s, b) u(s, b+h) w(s) d s \longrightarrow \int_{a}^{b} u^{2}(s, b) w(s) d s=1
$$

and plugging (35)-(38) into (33), then we obtain (32). The second part of the theorem follows from the above. 
Theorem 6 (eigenvalue-eigenfunction differential equation for separated BVPs). Let (22) hold. Consider the BVP (21), (23a)-(24b), with $0 \leq \alpha<\pi, 0<\beta \leq \pi$, that is, arbitrary separated conditions at $a$ and $b$. Using the notation of Section 2 and letting $\lambda=\lambda_{n}, u=u_{n}$, we have the following differential equations:

$$
\begin{aligned}
\lambda^{\prime}(b)= & p_{1}(b)\left(u^{\prime}(b, b)\right)^{2}+2 u^{[3]}(b, b) u^{\prime}(b, b) \\
& +\left(p_{2}(b)-\lambda(b) w(b)\right) u^{2}(b, b) \\
& -\frac{\left(u^{[2]}\right)^{2}(b, b)}{p_{0}(b)} \quad \text { a.e. in }(a, B) .
\end{aligned}
$$

Furthermore, if $\beta \neq \pi$, then

$$
\begin{aligned}
\lambda^{\prime}(b)= & p_{1}(b)\left(u^{\prime}(b, b)\right)^{2}+2 \cot \beta \mathrm{u}(\mathrm{b}, \mathrm{b}) \mathrm{u}^{\prime}(\mathrm{b}, \mathrm{b}) \\
& +\left(p_{2}(b)-\lambda(b) w(b)\right) u^{2}(b, b)-\frac{\left(u^{[2]}\right)^{2}(b, b)}{p_{0}(b)} .
\end{aligned}
$$

If $\beta \neq \pi / 2$, then

$$
\begin{aligned}
\lambda^{\prime}(b)= & p_{1}(b)\left(u^{\prime}(b, b)\right)^{2}+2 \tan \beta u^{[2]}(b, b) u^{[3]}(b, b) \\
& +\left(p_{2}(b)-\lambda(b) w(b)\right) u^{2}(b, b)-\frac{\left(u^{[2]}\right)^{2}(b, b)}{p_{0}(b)} .
\end{aligned}
$$

In particular, if $p_{0}, p_{1}$, and $p_{2}$ and $w$ are continuous at $b$ and $p_{0}(b) \neq 0$, then (39)-(41) hold at $b$.

Proof. The proof is more complicated but consists basically of combining the techniques in the proofs of Theorems 4 and 5 . For small $h$, we choose $\mu=\lambda(b), v=\lambda(b+h)$, and $u=u(\cdot, b)$, $v=u(\cdot, b+h)$. From (19) and the boundary conditions (23a)(24b), noting that $[u, v](a)=0$, we have

$$
\begin{aligned}
{[\lambda(b)-} & \lambda(b+h)] \int_{a}^{b} u(s, b) u(s, b+h) w(s) d s \\
= & u^{[3]}(b, b) u(b, b+h)-u^{[2]}(b, b) u^{\prime}(b, b+h) \\
& +u^{\prime}(b, b) u^{[2]}(b, b+h)-u(b, b) u^{[3]}(b, b+h) .
\end{aligned}
$$

Now dividing (42) by $h$ and taking the limit as $h \rightarrow 0$, plugging (28), (30), (35), and (36) into (42), and using the continuity of $\lambda$ at $b$, the uniform convergence of $u(\cdot, b+h)$ to $u(\cdot, b)$, and Lemma 3 , we obtain (39). In addition, from the boundary conditions (24a) $-(24 b)$ we note that if $\beta \neq \pi$ then $y^{[3]}(b)=\cot \beta y(b)$ and if $\beta \neq \pi / 2$ then $y^{[1]}(b)=\tan \beta y^{[2]}(b)$; plugging them into (39) we obtain (40) and (41).

It is easy to see that Theorem 6 includes Theorems 4 and 5.
Theorem 7. Let (22) hold. Fix a and consider the fourth-order Dirichlet eigenvalues $\lambda_{n}^{D}(b)=\lambda_{n}(b)(0, \pi ; a, b)$ for $b$ in $(a, B)$ defined as in (16). If

$$
p_{0} \geq 0 \text { a.e., } \quad \frac{p_{2}^{2}}{w} \in L_{\mathrm{loc}}(A, B),
$$

then, for $n \in N_{0}, \lambda_{n}(b)$ is strictly decreasing on $(a, B)$ and

$$
\lambda_{n}^{D}(b) \longrightarrow+\infty \quad \text { as } b \longrightarrow a^{+} .
$$

Proof. The decreasing property of $\lambda_{n}^{D}$ as a function of $b$ follows directly from Theorem 4 . Assume (44) is false, and then by Theorem $4 \lambda(b)=\lambda_{0}^{D}(b)$ has a finite limit, say $\lambda^{+}(a)$, as $b \rightarrow a^{+}$and hence is bounded on $\left(a, B_{1}\right)$ for $B_{1}<B$. Let $u=u_{0}(\cdot, b)$ be an eigenfunction of $\lambda(b)$ normalized to satisfy

$$
\int_{a}^{b} u^{2} w=1
$$

Next we show that

$$
\left(p_{0} u^{\prime \prime}\right)^{\prime}(a, b) \longrightarrow 0 \quad \text { as } b \longrightarrow a^{+} .
$$

To see this, we first show there exists at least one point $c \in$ $(a, b)$ such that $\left(p_{0} u^{\prime \prime}\right)^{\prime}(c, b)=0$. Noting that $u(a, b)=$ $u(b, b)=0$ and according to the Rolle's theorem we know, there exists at least one point $\xi_{0} \in(a, b)$ such that $u^{\prime}\left(\xi_{0}, b\right)=$ 0 . Similarly, noting that $u^{\prime}(a, b)=u^{\prime}(b, b)=0$, hence there exist at least two points $\xi_{1} \in\left(a, \xi_{0}\right)$ and $\xi_{2} \in\left(\xi_{0}, b\right)$, such that $\left(p_{0} u^{\prime \prime}\right)\left(\xi_{1}, b\right)=\left(p_{0} u^{\prime \prime}\right)\left(\xi_{2}, b\right)$. Therefor there exists at least one point $c \in\left(\xi_{1}, \xi_{2}\right)$, such that $\left(p_{0} u^{\prime \prime}\right)^{\prime}(c, b)=0$. Using $\left(p_{0} u^{\prime \prime}\right)^{\prime}(c, b)=0$, the boundedness of $\lambda$ and the Schwarz inequality, we get

$$
\begin{aligned}
& {\left[\left(p_{0} u^{\prime \prime}\right)^{\prime}(a, b)\right]^{2}} \\
& =\left[\left(p_{0} u^{\prime \prime}\right)^{\prime}(c, b)-\left(p_{0} u^{\prime \prime}\right)^{\prime}(a, b)\right]^{2} \\
& \quad=\left[\int_{a}^{c}\left(p_{0} u^{\prime \prime}\right)^{\prime \prime}\right]^{2} \\
& \quad=\left[\int_{a}^{c}\left(p_{1} u^{\prime}\right)^{\prime}-\left(p_{2}-\lambda w\right) u\right]^{2} \\
& \quad=\left[\left(p_{1} u^{\prime}\right)(c, b)-\int_{a}^{c}\left(p_{2}-\lambda w\right) u\right]^{2} \\
& \quad \leq 2\left[\left(p_{1} u^{\prime}\right)(c, b)\right]^{2}+2\left[\int_{a}^{c}\left(p_{2}-\lambda w\right) u\right]^{2}, \\
& {\left[\int_{a}^{c}\left(p_{2}-\lambda w\right) u\right]^{2}} \\
& \quad=\left[\int_{a}^{c}\left(p_{2} w^{-1 / 2}-\lambda w^{1 / 2}\right) w^{1 / 2} u\right]^{2} \\
& \quad \leq \int_{a}^{c}\left(p_{2} w^{-1 / 2}-\lambda w^{1 / 2}\right)^{2} \int_{a}^{c} u^{2} w \\
& \leq \int_{a}^{b}\left(\frac{p_{2}^{2}}{w}-2 \lambda p_{2}+\lambda^{2} w\right) \int_{a}^{b} u^{2} w \longrightarrow 0 \text { as } b \longrightarrow a^{+} .
\end{aligned}
$$


So

$$
\left[\left(p_{0} u^{\prime \prime}\right)^{\prime}(a, b)\right]^{2} \longrightarrow 0 \quad \text { as } b \longrightarrow a^{+}
$$

For $p_{0} u^{\prime \prime}(a, b)$, we have

$$
\begin{aligned}
& {\left[\left(p_{0} u^{\prime \prime}\right)(a, b)\right]^{2}} \\
& =\left[\left(p_{0} u^{\prime \prime}\right)\left(\xi_{1}, b\right)-\left(p_{0} u^{\prime \prime}\right)(a, b)\right]^{2} \\
& =\left[\int_{a}^{\xi_{1}}\left(p_{0} u^{\prime \prime}\right)^{\prime}\right]^{2} \\
& =\left[\int_{a}^{\xi_{1}} \int_{t}^{c}\left(p_{1} u^{\prime}\right)^{\prime}-\left(p_{2}-\lambda w\right) u d \xi d t\right]^{2} \\
& \leq 2\left[\int_{a}^{\xi_{1}} \int_{t}^{c}\left(p_{1} u^{\prime}\right)^{\prime} d \xi d t\right]^{2}+2\left[\int_{a}^{\xi_{1}} \int_{t}^{c}\left(p_{2}-\lambda w\right) u d \xi d t\right]^{2} \\
& =2\left[\int_{a}^{\xi_{1}}\left(p_{1} u^{\prime}\right)(c)-\left(p_{1} u^{\prime}\right)(t) d t\right]^{2} \\
& +2\left[\int_{a}^{\xi_{1}} \int_{t}^{c}\left(p_{2}-\lambda w\right) u d \xi d t\right]^{2} \\
& {\left[\int_{a}^{\xi_{1}} \int_{t}^{c}\left(p_{2}-\lambda w\right) u d \xi d t\right]^{2}} \\
& \leq(b-a) \int_{a}^{\xi_{1}}\left[\int_{t}^{c}\left(p_{2} w^{-1 / 2}-\lambda w^{1 / 2}\right)^{2} d \xi \int_{t}^{c} u^{2} w d \xi\right] d t \\
& \leq(b-a)^{2} \int_{a}^{b}\left(\frac{p_{2}^{2}}{w}-2 \lambda p_{2}+\lambda^{2} w\right) \\
& \times \int_{a}^{b} u^{2} w \longrightarrow 0 \text { as } b \longrightarrow a^{+} .
\end{aligned}
$$

Thus

$$
\left[\left(p_{0} u^{\prime \prime}\right)(a, b)\right]^{2} \longrightarrow 0 \quad \text { as } b \longrightarrow a^{+}
$$

Noting that $\lambda(b) \rightarrow \lambda^{+}(a)$ as $b \rightarrow a^{+}$, by (46) and the continuous dependence of solutions (21) on initial conditions and on the parameter we conclude that $u(\cdot, b) \rightarrow 0$ uniformly on any compact subinterval of $[a, B)$. Therefore, for $\varepsilon>0$, there exists a $b_{0} \in(a, B)$, such that

$$
|u(t, b)|<\varepsilon, \quad t \in[a, b], a<b<b_{0} .
$$

This implies that

$$
\int_{a}^{b} u^{2} w<\varepsilon^{2} \int_{a}^{b} w
$$

for $\varepsilon$ sufficiently small. This contradicts the normalization (45), which completes the proof.

\section{Eigenvalues of Higher-Order Sturm-Liouville Problem}

In this section, we obtain the differentiability of the eigenvalues of the $2 k$ th-order boundary value problem and establish differential equations satisfied by them and discuss the behavior of $2 k$ th-order Dirichlet eigenvalueas functions of the endpoint $b$.

Theorem 8 ( $2 k$ th-order Dirichlet eigenvalue-eigenfunction differential equation). Let (7) hold. Consider the BVP (6), (13a)-(14c), with $0 \leq \alpha<\pi$ and $\beta=\pi$, that is, arbitrary separated conditions at $a$ and the $2 k$ th-order Dirichlet conditions at $b$. Using the notation of Section 2 and letting $\lambda=\lambda_{n}, u=u_{n}$, we have the following differential equation:

$$
\left(p_{0} \lambda^{\prime}\right)(b)=-\left(u^{[k]}\right)^{2}(b, b) \quad \text { a.e. in }(a, B) .
$$

In particular, if $p_{0}$ is continuous at $b \in[a, B)$ and $p_{0}(b) \neq 0$, then (53) holds at $b$.

Proof. For small $h$, in (19), choose $\mu=\lambda(b), \nu=\lambda(b+h)$, and $u=u(\cdot, b), v=u(\cdot, b+h)$. From (19) and the boundary conditions (13a)-(14c), noting that $[u, v](a)=0, u^{[j]}(b, b)=$ $0(j=0,1,2, \ldots, k-1)$, we have

$$
\begin{aligned}
& (-1)^{k} \sum_{r=k}^{2 k-1}(-1)^{2 k+1-r} u^{[r]}(b, b) u^{[2 k-r-1]}(b, b+h) \\
& \quad=[\lambda(b)-\lambda(b+h)] \int_{a}^{b} u(s, b) u(s, b+h) w(s) d s .
\end{aligned}
$$

Hence

$$
\begin{aligned}
& u^{[r]}(b, b+h) \\
& \quad=u^{(r)}(b, b+h)-u^{(r)}(b+h, b+h) \\
& \quad=-\int_{b}^{b+h} u^{(r+1)}(s, b+h) d s, \quad r=0,1, \ldots, k-2 .
\end{aligned}
$$

So by Lemmas 1 and 3, we have

$$
\lim _{h \rightarrow 0} \frac{u^{(r)}(b, b+h)}{h}=-u^{(r+1)}(b, b), \quad r=0,1, \ldots, k-2 .
$$

Similarly, from

$$
\begin{aligned}
u^{(k-1)}(b, b+h) & =u^{(k-1)}(b, b+h)-u^{(k-1)}(b+h, b+h) \\
& =-\int_{b}^{b+h} u^{(k)}(s, b+h) d s \\
& =-\int_{b}^{b+h} \frac{\left(p_{0} u^{(k)}\right)(s, b+h)}{p_{0}(s)} d s,
\end{aligned}
$$


we can have

$$
\lim _{h \rightarrow 0} \frac{u^{(k-1)}(b, b+h)}{h}=-\frac{1}{p_{0}(b)}\left(p_{0} u^{(k)}\right)(b, b) .
$$

In addition, noting that

$$
\int_{a}^{b} u(s, b) u(s, b+h) w(s) d s \longrightarrow \int_{a}^{b} u^{2}(s, b) w(s) d s=1
$$$$
\text { as } h \longrightarrow 0 \text {. }
$$

Plugging (56), (58), and (59) into (54) divided by $h$ and taking the limit as $h \rightarrow 0$, we get (53). The second part of the theorem follows from above.

Theorem 9 ( $2 k$ th-order Neumann eigenvalue-eigenfunction differential equation). Let (7) hold. Consider the BVP (6), (13a)-(14c), with $0 \leq \alpha<\pi$ and $\beta=\pi / 2$, that is, arbitrary separated conditions at $a$ and the $2 k$ th-order Neumann conditions at $b$. Using the notation of Section 2 and letting $\lambda=\lambda_{n}, u=u_{n}$, we have the following differential equation:

$$
\begin{aligned}
\lambda^{\prime}(b)= & \sum_{r=1}^{k-1} p_{k-r}(b)\left(u^{(r)}(b, b)\right)^{2} \\
& +\left(p_{k}(b)-\lambda(b) w(b)\right) u^{2}(b, b) \text { a.e. in }(a, B) .
\end{aligned}
$$

In particular, if $p_{1}, p_{2}, \ldots, p_{k}$ and $w$ are continuous at $b \in$ $[a, B)$, then (60) holds at $b$.

Proof. The proof is similar to Theorem 8 . For small $h$, we choose $\mu=\lambda(b), v=\lambda(b+h)$, and $u=u(\cdot, b), v=u(\cdot, b+h)$. From (19) and the boundary conditions (13a)-(14c), noting that $[u, v](a)=0, u^{[j]}(b, b)=0, j=k, k+1, \ldots, 2 k-1$, we have

$$
\begin{aligned}
& (-1)^{k} \sum_{r=0}^{k-1}(-1)^{2 k+1-r} u^{[r]}(b, b) u^{[2 k-r-1]}(b, b+h) \\
& \quad=[\lambda(b)-\lambda(b+h)] \int_{a}^{b} u(s, b) u(s, b+h) w(s) d s \\
& (-1)^{k}\left(u^{[2 k-1]}\right)(b, b+h) \\
& =(-1)^{k} u^{[2 k-1]}(b, b+h)-(-1)^{k} u^{[2 k-1]}(b+h, b+h) \\
& =-\int_{b}^{b+h}(-1)^{k}\left(u^{[2 k-1]}\right)^{\prime}(s, b+h) d s \\
& =-\int_{b}^{b+h}(-1)^{k}\left(u^{[2 k]}-(-1)^{k} p_{k} u\right)(s, b+h) d s
\end{aligned}
$$

$$
\begin{aligned}
= & \int_{b}^{b+h}\left[p_{k}(s) u(s, b+h)-\lambda(b+h) u(s, b+h) w(s)\right] d s \\
= & \int_{b}^{b+h} p_{k}(s) u(s, b) d s \\
& +\int_{b}^{b+h} p_{k}(s) u(s, b+h)-p_{k}(s) u(s, b) d s \\
& -\lambda(b+h) \int_{b}^{b+h} u(s, b) w(s) d s \\
& +\lambda(b+h) \int_{b}^{b+h}[u(s, b)-u(s, b+h)] w(s) d s \\
= & \int_{b}^{b+h} p_{k}(s) u(s, b) d s-\lambda(b+h) \int_{b}^{b+h} u(s, b) w(s) d s .
\end{aligned}
$$

By Lemmas 1 and 3 we have

$$
\begin{aligned}
\lim _{h \rightarrow 0} & \frac{(-1)^{k}\left(u^{[2 k-1]}\right)(b, b+h)}{h} \\
& =\left(p_{k}(b)-\lambda(b) w(b)\right) u(b, b) .
\end{aligned}
$$

In a similar way, we have

$$
\begin{aligned}
\lim _{h \rightarrow 0} \frac{(-1)^{k}\left(u^{[2 k-r-1]}\right)(b, b+h)}{h} \\
=(-1)^{k+1}\left(u^{[2 k-r-1]}\right)^{\prime}(b, b) .
\end{aligned}
$$

Combining $\left(u^{[2 k-r-1]}\right)^{\prime}=u^{[2 k-r]}-(-1)^{k-r} p_{k-r} y^{(r)}$, we also can get

$$
\begin{aligned}
\lim _{h \rightarrow 0} & \frac{(-1)^{k}\left(u^{[2 k-r-1]}\right)(b, b+h)}{h} \\
& =(-1)^{k+1} u^{[2 k-r]}(b, b)-(-1)^{2 k-r+1} p_{k-r} u^{(r)}(b, b) \\
& =-(-1)^{2 k-r+1} p_{k-r} u^{(r)}(b, b), \quad r=1,2, \ldots, k-1 .
\end{aligned}
$$

When $h \rightarrow 0$, noting that

$$
\int_{a}^{b} u(s, b) u(s, b+h) w(s) d s \longrightarrow \int_{a}^{b} u^{2}(s, b) w(s) d s=1
$$

and plugging (63)-(66) into (61), then we obtain (60). The second part of the theorem follows from the above.

Theorem 10 (eigenvalue-eigenfunction differential equation for separated BVPs). Let (7) hold. Consider the BVP (6), (13a)-(14c), with $0 \leq \alpha<\pi, 0<\beta \leq \pi$, that is, arbitrary separated conditions at $a$ and $b$. Using the notation of Section 2 
and letting $\lambda=\lambda_{n}, u=u_{n}$, we have the following differential equations:

$$
\begin{aligned}
\lambda^{\prime}(b)=\sum_{r=1}^{k-1} & {\left[p_{k-r}(b)\left(u^{[r]}(b, b)\right)^{2}+2 u^{[2 k-r]}(b, b) u^{[r]}(b, b)\right] } \\
& +\left(p_{k}(b)-\lambda(b) w(b)\right) u^{2}(b, b) \\
& -\frac{\left(u^{[k]}\right)^{2}(b, b)}{p_{0}(b)} \quad \text { a.e. in }(a, B) .
\end{aligned}
$$

Furthermore, if $\beta \neq \pi$, then

$$
\begin{aligned}
& \lambda^{\prime}(b) \\
& =\sum_{r=1}^{k-1}\left[p_{k-r}(b)\left(u^{[r]}(b, b)\right)^{2}+2 \cot \beta \mathrm{u}^{[\mathrm{r}-1]}(\mathrm{b}, \mathrm{b}) \mathrm{u}^{[\mathrm{r}]}(\mathrm{b}, \mathrm{b})\right] \\
& \quad+\left(p_{k}(b)-\lambda(b) w(b)\right) u^{2}(b, b)-\frac{\left(u^{[k]}\right)^{2}(b, b)}{p_{0}(b)} .
\end{aligned}
$$

If $\beta \neq \pi / 2$, then

$$
\begin{aligned}
& \lambda^{\prime}(b) \\
& =\sum_{r=1}^{k-1}\left[p_{k-r}(b)\left(u^{[r]}(b, b)\right)^{2}\right. \\
& \left.+2 \tan \beta u^{[2 k-r]}(b, b) u^{[2 k-r+1]}(b, b)\right] \\
& +\left(p_{k}(b)-\lambda(b) w(b)\right) u^{2}(b, b)-\frac{\left(u^{[k]}\right)^{2}(b, b)}{p_{0}(b)} \text {. }
\end{aligned}
$$

In particular, if $p_{0}, p_{1}, \ldots, p_{k}$ and $w$ are continuous at $b$ and $p_{0}(b) \neq 0$, then (67)-(69) hold at $b$.

Proof. The proof is more complicated but consists basically of combining the techniques in the proofs of Theorems 8 and 9. The concrete process is omitted. 9.

It is easy to see that Theorem 10 includes Theorems 8 and

Theorem 11. Let (7) hold. Fix $a$ and consider the $2 k$ th-order Dirichlet eigenvalues $\lambda_{n}^{D}(b)=\lambda_{n}(b)(0, \pi ; a, b)$ for $b$ in $(a, B)$ defined as in (16). If

$$
p_{0} \geq 0 \text { a.e. }, \quad \frac{p_{k}^{2}}{w} \in L_{\text {loc }}(A, B),
$$

then, for $n \in N_{0}, \lambda_{n}(b)$ is strictly decreasing on $(a, B)$ and

$$
\lambda_{n}^{D}(b) \longrightarrow+\infty \quad \text { as } b \longrightarrow a^{+} .
$$

Proof. The decreasing property of $\lambda_{n}^{D}$ as a function of $b$ follows directly from Theorem 8 . Assume (71) is false, and then by Theorem $8 \lambda(b)=\lambda_{0}^{D}$ has a finite limit, say $\lambda^{+}(a)$, as $b \rightarrow a^{+}$, and hence is bounded on $\left(a, B_{1}\right)$ for $B_{1}<B$. Let $u=u_{0}(\cdot, b)$ be an eigenfunction of $\lambda(b)$ normalized to satisfy

$$
\int_{a}^{b} u^{2} w=1
$$

First we show that

$$
\left(p_{0} u^{(k)}\right)^{(k-1)}(a, b) \longrightarrow 0 \quad \text { as } b \longrightarrow a^{+}
$$

To see this, we first show there exists at least one point $c \in$ $(a, b)$ such that $\left(p_{0} u^{(k)}\right)^{(k-1)}(c, b)=0$. Noting that $u^{(r)}(a, b)=$ $u^{(r)}(b, b)=0, r=0,1, \ldots, k-1$, and according to the Rolle's theorem we know, there exists at least one point $\xi_{0} \in(a, b)$ such that $u^{\prime}\left(\xi_{0}, b\right)=0$. In the same way, there exist at least $r$ points $\xi_{i} \in(a, b), \xi_{1}<\xi_{2}<\cdots<\xi_{r}$, such that $u^{(r)}\left(\xi_{i}, b\right)=0$, $i=1,2, \ldots, r, r=1,2, \ldots, k-1$. So there exist at least $k$ points $\eta_{s} \in\left(\xi_{r-1}, \xi_{r}\right), r=1,2, \ldots, k, \xi_{0}=a, \xi_{k}=b$, such that $\left(p_{0} u^{(k)}\right)\left(\eta_{s}, b\right)=0$. Thus by the Rolle's theorem we can get the conclusion that there exist at least $k-r$ points $\zeta_{k-r}$, such that $\left(p_{0} u^{(k)}\right)^{r}\left(\zeta_{k-r}, b\right)=0, r=0,1, \ldots, k-1$. In addition using the boundedness of $\lambda$ and the Schwarz inequality, we get

$$
\begin{aligned}
& {\left[\left(p_{0} u^{(k)}\right)^{(k-1)}(a, b)\right]^{2}} \\
& =\left[\left(p_{0} u^{(k)}\right)^{(k-1)}(c, b)-\left(p_{0} u^{(k)}\right)^{(k-1)}(a, b)\right]^{2} \\
& =\left[\int_{a}^{c}\left(p_{0} u^{(k)}\right)^{(k)}\right]^{2} \\
& =\left[\int_{a}^{c} \sum_{r=1}^{k-1}\left(p_{k-r} u^{(r)}\right)^{(r)}-\left(p_{k}-\lambda w\right) u\right]^{2} \\
& \leq 2\left[\int_{a}^{c} \sum_{r=1}^{k-1}\left(p_{k-r} u^{(r)}\right)^{(r)}\right]^{2}+2\left[\int_{a}^{c}\left(p_{k}-\lambda w\right) u\right]^{2} \\
& \leq 2\left[\sum_{r=1}^{k-1}(b-a)^{r-1}\left(p_{k-r} u^{(r)}\right)(t, b)\right]^{2} \\
& +2\left[\int_{a}^{c}\left(p_{k}-\lambda w\right) u\right]^{2} \\
& {\left[\int_{a}^{c}\left(p_{k}-\lambda w\right) u\right]^{2}} \\
& =\left[\int_{a}^{c}\left(p_{k} w^{-1 / 2}-\lambda w^{1 / 2}\right) w^{1 / 2} u\right]^{2} \\
& \leq \int_{a}^{c}\left(p_{k} w^{-1 / 2}-\lambda w^{1 / 2}\right)^{2} \int_{a}^{c} u^{2} w \\
& \leq \int_{a}^{b}\left(\frac{p_{k}^{2}}{w}-2 \lambda p_{k}+\lambda^{2} w\right) \int_{a}^{b} u^{2} w \longrightarrow 0 \text { as } b \longrightarrow a^{+} \text {. }
\end{aligned}
$$


So

$$
\left[\left(p_{0} u^{(k)}\right)^{(k-1)}(a, b)\right]^{2} \longrightarrow 0 \quad \text { as } b \longrightarrow a^{+}
$$

Next we show that

$$
\left(p_{0} u^{(k)}\right)^{(k-r)}(a, b) \longrightarrow 0, \quad r=2,3, \ldots, k, \text { as } b \longrightarrow a^{+} .
$$

For $i=1,2, \ldots, k-1, i \geq j$, also according to the Rolle's theorem we know, there exist at least $i-j$ zero points such that $\left(p_{k-i} u^{(i)}\right)^{(j)}(\cdot, b)=0$. Let $c_{r}$ be the first zero point of $\left(p_{0} u^{(k)}\right)^{(k-r)}(\cdot, b)=0$. For $\left(p_{0} u^{(k)}\right)^{(k-r)}(a, b)$, we have

$$
\begin{aligned}
{\left[\left(p_{0} u^{(k)}\right)^{(k-r)}(a, b)\right]^{2} } & \\
= & {\left[\left(p_{0} u^{(k)}\right)^{(k-r)}\left(c_{r}, b\right)-\left(p_{0} u^{(k)}\right)^{(k-r)}(a, b)\right]^{2} } \\
= & {\left[\int_{a}^{c_{r}}\left(p_{0} u^{(k)}\right)^{(k-r+1)}\right]^{2} } \\
= & {\left[\int_{a}^{c_{r}}\left(p_{0} u^{(k)}\right)^{(k-r+1)}\left(c_{r+1}, b\right)-\left(p_{0} u^{(k)}\right)^{(k-r+1)}\left(t_{r+1}, b\right)\right]^{2} } \\
= & {\left[\int_{a}^{c_{r}} \int_{t_{r+1}}^{c_{r+1}}\left(p_{0} u^{(k)}\right)^{(k-r+2)}\right]^{2} } \\
= & \cdots=\left[\int_{a}^{c_{r}} \int_{t_{r+1}}^{c_{r+1}} \ldots \int_{t_{k}}^{c_{k}}\left(p_{0} u^{(k)}\right)^{(k)}\right]^{2} \\
= & {\left[\int_{a}^{c_{r}} \int_{t_{r+1}}^{c_{r+1}} \ldots \int_{t_{k}}^{c_{k}} \sum_{i=1}^{k-1}\left(p_{k-i} u^{(i)}\right)^{(i)}\right.} \\
& +2(b-a)^{2(k-r)}\left[\int_{t_{k}}^{c_{k}}\left(p_{k}-\lambda w\right) u d \xi_{k}\right]^{2}, \\
& +2(b-a)^{2(k-r)}\left[\int_{t_{k}}^{c_{k}}\left(p_{k}-\lambda w\right) u d \xi_{k}\right]^{2}(b-a)^{2(k-r+i)}\left[\int_{t_{k}}^{c_{k}} p_{k-i} u^{(i)}\right]^{2} \\
\leq & 2\left[\int_{a}^{c_{r}} \int_{t_{r+1}}^{c_{r+1}} \ldots \int_{t_{k}}^{c_{k}} \sum_{i=1}^{c_{k}-1}\left(p_{k-i} u^{(i)}\right)^{(i)} d \xi_{k} \cdots d \xi_{r+1} d t\right]^{2} \\
& +2\left[\int_{a}^{c_{r}} \int_{t_{r+1}}^{c_{r+1}} \ldots \int_{t_{k}}^{c_{k}}\left(p_{k}-\lambda w\right) u d \xi_{k} \cdots d \xi_{r+1} d t\right]^{2}
\end{aligned}
$$

$$
\begin{aligned}
& {\left[\int_{t_{k}}^{c_{k}} p_{k-i} u^{(i)}\right]^{2}} \\
& \quad \leq(b-a)^{2}\left|p_{k-i} u^{(i)}(t)\right|^{2} \\
& \quad \longrightarrow 0 \text { as } b \longrightarrow a^{+}, t \in(a, b), i=1,2, \ldots, k-1, \\
& \quad\left[\int_{t_{k}}^{c_{k}}\left(p_{k}-\lambda w\right) u d \xi\right]^{2} \\
& \quad \leq\left[\int_{t_{k}}^{c_{k}}\left(p_{k} w^{-1 / 2}-\lambda w^{1 / 2}\right)^{2} d \xi \int_{t}^{c} u^{2} w d \xi\right] \\
& \quad \leq \int_{a}^{b}\left(\frac{p_{k}^{2}}{w}-2 \lambda p_{k}+\lambda^{2} w\right) \int_{a}^{b} u^{2} w \longrightarrow 0 \quad \text { as } b \longrightarrow a^{+} .
\end{aligned}
$$

Thus

$$
\left[\left(p_{0} u^{(k)}\right)^{(k-r)}(a, b)\right]^{2} \longrightarrow 0, \quad r=2,3, \ldots, k, \text { as } b \longrightarrow a^{+} .
$$

Noting that $\lambda(b) \rightarrow \lambda^{+}(a)$ as $b \rightarrow a^{+}$, by (73), (78) and the continuous dependence of solutions (6) on initial conditions and on the parameter we conclude that $u(\cdot, b) \rightarrow 0$ uniformly on any compact subinterval of $[a, B)$. Therefore, for $\varepsilon>0$, there exists a $b_{0} \in[a, B)$, such that

$$
|u(t, b)|<\varepsilon, \quad t \in[a, b], a<b<b_{0} .
$$

This implies that

$$
\int_{a}^{b} u^{2} w<\varepsilon^{2} \int_{a}^{b} w
$$

for $\varepsilon$ sufficiently small. This contradicts the normalization (72), which completes the proof.

\section{Conclusion}

With a simple analysis, we showed that the eigenvalues of a class of $2 k$ th-order Sturm-Liouville problems depend not only continuously but also smoothly on boundary points and that the derivative of the $n$th eigenvalue as a function of an endpoint satisfies a first order differential equation. It is satisfying that these equations are established without any smoothness assumptions on the coefficients and also for the case that the leading coefficient $p_{0}$ is not assumed to be bounded away from zero and is even allowed to change sign. More importantly, we show that the lowest Dirichlet eigenvalue is a decreasing function of the endpoints and has an infinite limit as the endpoints approach each other.

In recent years, the various physics applications of this kind Sturm-Liouville problem are found in much literature (see, e.g., [11-15]). Many topics in mathematical physics require the investigation of the eigenvalues and eigenfunctions of Sturm-Liouville-type boundary value problems. Our results contain all the cases when $k$ is equal to certain special positive integer. In particular, for $k=2$, Theorem 11 explains 
that natural frequency of the rod will increase with the shortening of its length.

Furthermore, highly important results in this field have been obtained for the case when the eigenparameter appears not only in the differential equation with transmission conditions but also in the boundary conditions. Particularly, on computing eigenvalues of these types Sturm-Liouville problems, we can refer to [16-18]. Therefore, our proof methods and results will be useful to resolve eigenvalue problem of discontinuous Sturm-Liouville operators and differential operators with eigenparameter boundary conditions.

\section{Conflict of Interests}

The authors declare that there is no conflict of interests regarding the publication of this paper.

\section{Acknowledgments}

The authors thank the referee for his/her careful reading of the paper and for making suggestions which have improved the presentation of the paper. The work of the first and third authors is supported by the Talent Introduction Project of Dezhou University (Grant no. 311694) and the work of the second author is supported by the National Nature Science Foundation of China (Grant no. 11361039).

\section{References}

[1] M. Dauge and B. Helffer, "Eigenvalues variation. I. Neumann problem for Sturm-Liouville operators," Journal of Differential Equations, vol. 104, no. 2, pp. 243-262, 1993.

[2] M. Dauge and B. Helffer, "Eigenvalues variation, II. Neumann problem for Sturm-Liouville operators," Journal of Differential Equations, vol. 104, pp. 263-297, 1993.

[3] Q. Kong and A. Zettl, "Dependence of eigenvalues of SturmLiouville problems on the boundary," Journal of Differential Equations, vol. 126, no. 2, pp. 389-407, 1996.

[4] S. Q. Ge, W. Y. Wang, and J. Q. Suo, "Dependence of eigenvalues of a class of fourth-order Sturm-Liouville problems on the boundary," Applied Mathematics and Computation, vol. 220, pp. 268-276, 2013.

[5] Q. Kong and A. Zettl, "Eigenvalues of regular Sturm-Liouville problems," Journal of Differential Equations, vol. 131, no. 1, pp. $1-19,1996$.

[6] Q. Kong, H. Wu, and A. Zettl, "Dependence of the $n$th SturmLiouville eigenvalue on the problem," Journal of Differential Equations, vol. 156, no. 2, pp. 328-354, 1999.

[7] Q. Kong, H. Wu, and A. Zettl, "Geometric aspects of SturmLiouville problems. I. structures on spaces of boundary conditions," Proceedings of the Royal Society of Edinburgh Section A: Mathematics, vol. 130, no. 3, pp. 561-589, 2000.

[8] X. Cao, Q. Kong, H. Wu, and A. Zettl, "Sturm-Liouville problems whose leading coefficient function changes sign," Canadian Journal of Mathematics, vol. 55, no. 4, pp. 724-749, 2003.

[9] Q. Kong, H. Wu, and A. Zettl, "Limits of Sturm-Liouville eigenvalues when the interval shrinks to an end point," Proceedings of the Royal Society of Edinburgh Section A: Mathematics, vol. 138, no. 2, pp. 323-338, 2008.
[10] A. P. Wang, J. Sun, and A. Zettl, “The classification of self-adjoint boundary conditions: separated, coupled, and mixed," Journal of Functional Analysis, vol. 255, no. 6, pp. 1554-1573, 2008.

[11] M. Demirci, Z. Akdoğan, and O. Sh. Mukhtarov, "Asymptotic behavior of eigenvalues and eigenfuctions of one discontinuous boundary value problem," International Journal of Computational Cogonition, vol. 2, no. 3, pp. 101-113, 2004.

[12] D. Buschmann, G. Stolz, and J. Weidmann, "One-dimensional Schrödinger operators with local point interactions," Journal für die Reine und Angewandte Mathematik, vol. 467, pp. 169-186, 1995.

[13] E. H. Doha, A. H. Bhrawy, and R. M. Hafez, "A JacobiJacobi dual-Petrov-Galerkin method for third- and fifth-order differential equations," Mathematical and Computer Modelling, vol. 53, no. 9-10, pp. 1820-1832, 2011.

[14] E. H. Doha, A. H. Bhrawy, and R. M. Hafez, "A Jacobi dualPetrov-Galerkin method for solving some odd-order ordinary differential equations," Abstract and Applied Analysis, vol. 2011, Article ID 947230, 21 pages, 2011.

[15] E. Tohidi, A. H. Bhrawy, and K. Erfani, "A collocation method based on Bernoulli operational matrix for numerical solution of generalized pantograph equation," Applied Mathematical Modelling. Simulation and Computation for Engineering and Environmental Systems, vol. 37, no. 6, pp. 4283-4294, 2013.

[16] M. M. Tharwat, A. H. Bhrawy, and A. S. Alofi, "Computing eigenvalues of discontinuous Sturm-Liouville problems with eigenparameter in all boundary conditions using Hermite approximation," Abstract and Applied Analysis, vol. 2013, Article ID 498457, 14 pages, 2013.

[17] M. M. Tharwat, A. H. Bhrawy, and A. Yildirim, "Numerical computation of eigenvalues of discontinuous Sturm-Liouville problems with parameter dependent boundary conditions using sinc method," Numerical Algorithms, vol. 63, no. 1, pp. 2748, 2013.

[18] M. M. Tharwat, A. H. Bhrawy, and A. S. Alofi, "Approximation of eigenvalues of discontinuous Sturm-Liouville problems with eigenparameter in all boundary conditions," Boundary Value Problems, vol. 2013, article 132, 2013. 


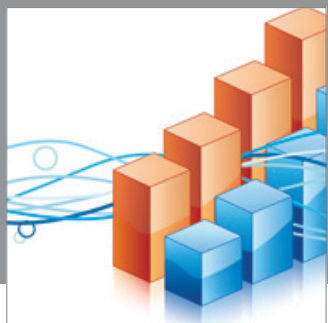

Advances in

Operations Research

mansans

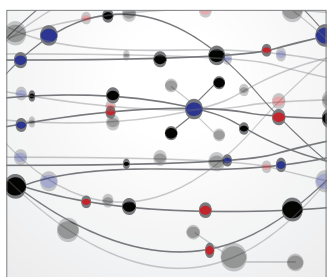

The Scientific World Journal
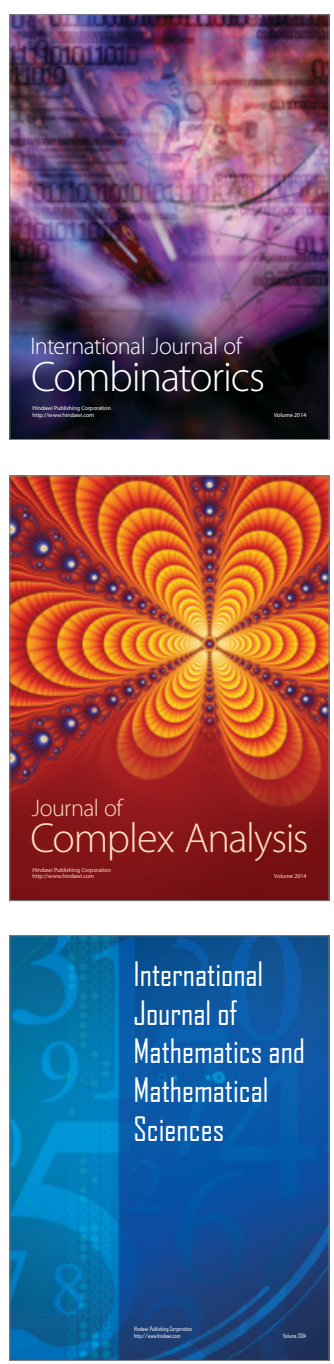
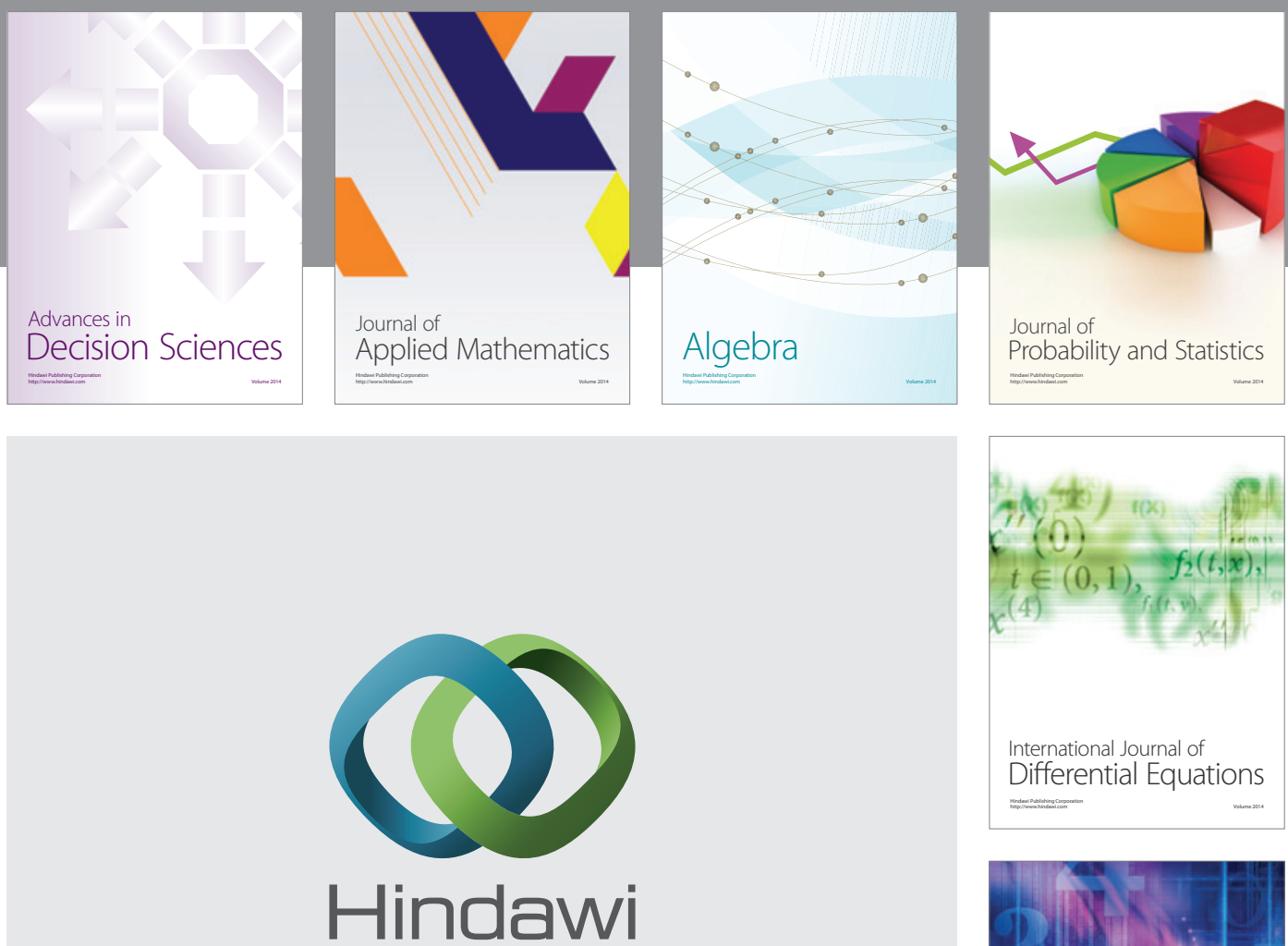

Submit your manuscripts at http://www.hindawi.com
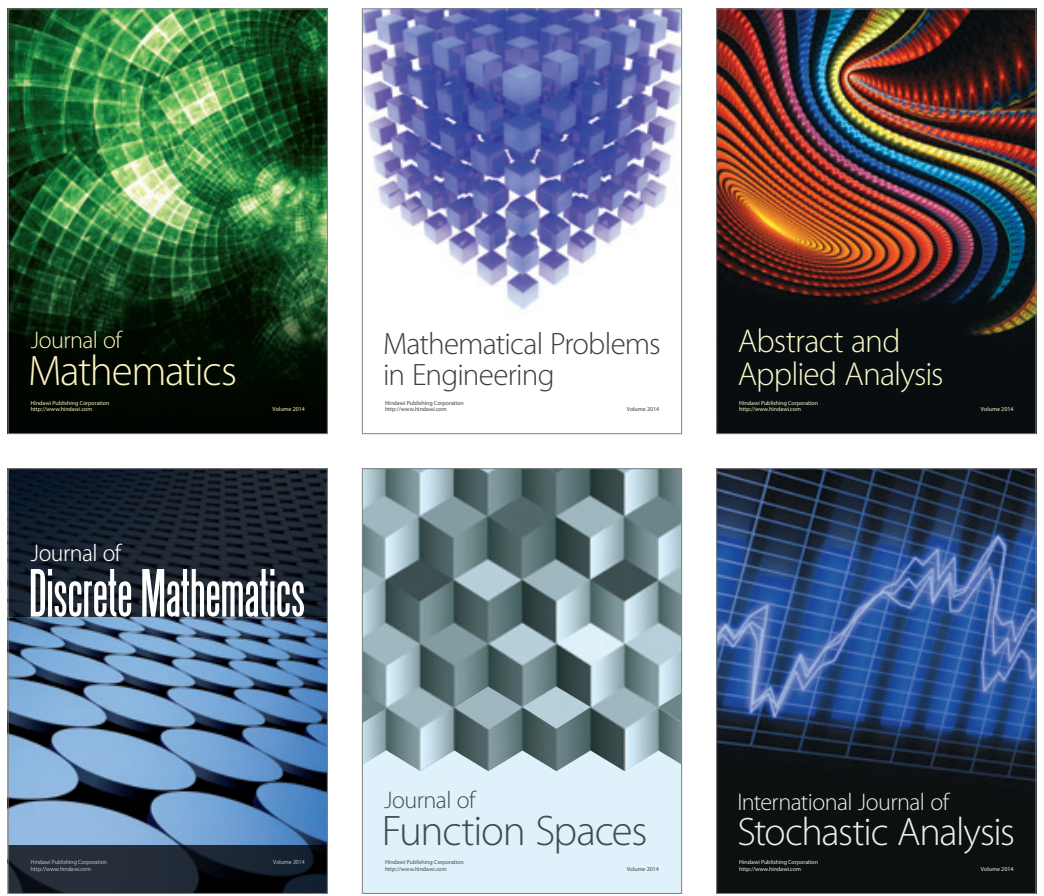

Journal of

Function Spaces

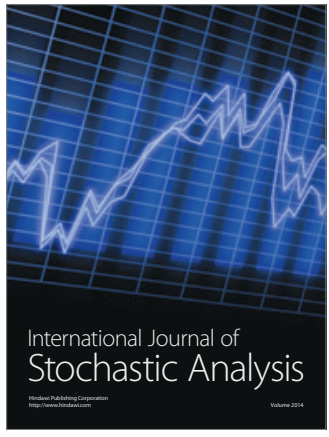

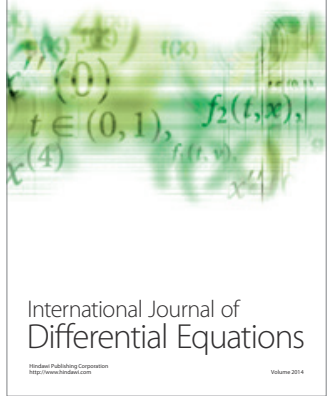
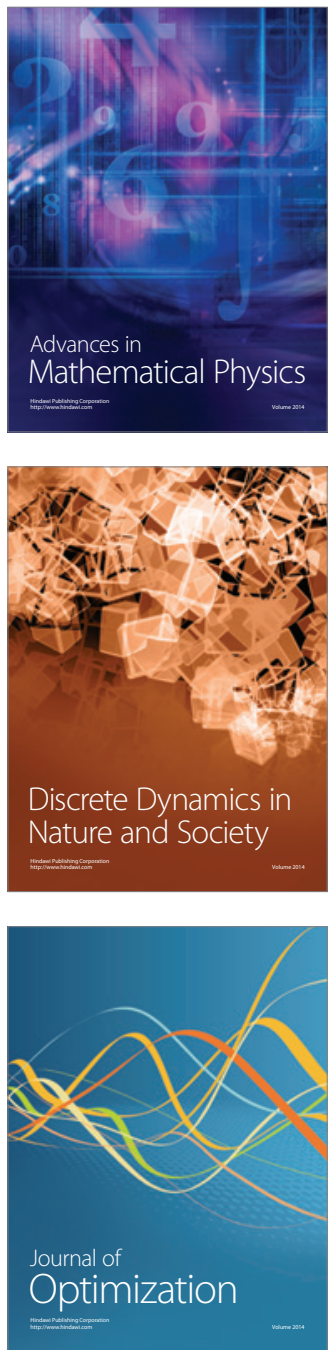